\title{
Modification of physicochemical and mechanical properties of a new bio-based gelatin composite films through composition adjustment and instantizing process
}

\begin{abstract}
The production of a new bio-based bovine gelatin (BG) film with incorporation of pea protein isolate (PPI), psyllium gum (PSY) and tragacanth (TRA) gum, and plasticized with glycerol, was completed via a two-stage fluidized bed agglomeration and casting techniques. Fluidization technique was used as a novel method to prepare instant film-forming powder (IFFP) in order to improve the films' physicochemical, mechanical and handling properties. The incorporation of PSY, TRA, and PPI greatly improved the physicochemical, thermal and mechanical properties of the film as compared to control films. Composite films containing high glycerol concentration displayed significant $(\mathrm{p}<0.05)$ reductions in tensile strength $(\mathrm{TS})$ and melting point (Tm) values. The FTIR and DSC results showed that PSY, TRA and PPI interacted effectively with gelatin. In addition, scanning electron microscopy (SEM) showed film surfaces without any cracks. The results demonstrated the miscibility and compatibility of the employed plasticizer (glycerol), polysaccharides (PSY and TRA) and protein (PPI) in composite films. Gelatin-based composite film containing $0.65 \mathrm{~g}$ glycerol (w/w, based on $5 \mathrm{~g}$ IFFP), $15.0 \mathrm{~g}$ gelatin, $3.0 \mathrm{~g}$ PPI, and $1.0 \mathrm{~g}$ of TRA/PSY (30:70 ratio, $\mathrm{mL} / \mathrm{mL}$ ) possessed the best characteristics in terms of MC, TS, Tm, and swelling properties as determined by response surface methodology.
\end{abstract}

Keyword: Optimization; Response surface methodology; Psyllium gum; Tragacanth gum; Pea protein isolate; Fluidized dryer 